\title{
Efecto nematicida y fungicida de extractos hidroalcohólicos de Piper lapathifolium y $\boldsymbol{P}$. melastomoides
}

Nematicidal and fungicidal effect of hydroalcoholic extracts of Piper lapathifolium and $P$. melastomoides

Carmona Hernández, Oscar ${ }^{1}$, José Armando Lozada García ${ }^{2 凶}$, María del Socorro Fernández ${ }^{2}$, Mauricio Luna Rodríguez ${ }^{1}$, María de Jesús Martínez Hernández y José Antonio Guerrero Analco ${ }^{3}$

${ }^{1}$ Facultad de Ciencias Agrícolas Xalapa, Universidad Veracruzana. Circuito Universitario Gonzalo Aguirre Beltrán s/n, Col. Zona Universitaria, Xalapa, Veracruz C.P. 91000

${ }^{2}$ Facultad de Biología Xalapa, Universidad Veracruzana. Circuito Universitario Gonzalo Aguirre Beltrán s/n, Col. Zona Universitaria, Xalapa, Veracruz C.P. 91000

${ }^{3}$ Red de Estudios Moleculares Avanzados, Instituto de Ecología A. C. Carretera Antigua Xalapa-Coatepec Km 2.5. Xalapa, Veracruz.

${ }^{凶}$ Autor de correspondencia: alozada@uv.mx

Recibido: $13 / 07 / 2018$

Aceptado: 12/09/2018

\section{RESUMEN}

El género Piper ha mostrado tener efectos biocidas sobre diferentes organismos nocivos para la agricultura como lo son nematodos y hongos fitopatógenos. Debido a que mayoría de estos organismos son contralados o erradicados con compuestos químicos tóxicos, se han estado buscando alternativas para sustituirlos, incluyendo el uso de extractos naturales, como los de Piper. En este sentido el presente trabajo se evaluó el efecto nematicida y fungicida de extractos hidroalcohólicos $\left(\right.$ ETOH: $\mathrm{H}_{2} \mathrm{O}$ 7:3) de Piper lapathifolium y P. melastomoides. El efecto nematicida se determinó en Panagrellus ridivivus en un ensayo a 24 horas y se estimó la Concentración Letal Media (CL50). El efecto fungicida se evaluó en Fusarium oxysporum y F. solani, por el ensayo de dilución en placa. La Concentración Efectiva Media ( $\mathrm{CE}_{50}$ ) se estableció mediante el modelo de Probit. Los resultados mostraron que $P$. melastomoides presentó efecto nematicida a concentraciones menores de $5 \mathrm{mg} / \mathrm{mL}$ con una CL50 de $2.32 \mathrm{mg} / \mathrm{mL}$, en el caso de $P$. lapathifolium no presentó actividad en los nematodos, en el caso de la actividad fungicida este extracto fue el que tuvo mayor actividad en ambas especies de Fusarium ( $\mathrm{CE}_{50}: 26.32$ y $5.83 \mathrm{mg} / \mathrm{mL}$ ), mientras que para $P$. melastomoides se presentó una menor actividad. Se concluye que $P$. melastomoides tiene efecto nematicida y P. lapathifolium fungicida.

Palabras clave: Fusarium, Panagrellus redivivus, Biocida.

\begin{abstract}
The Piper has been shown to have biocidal effects on different organisms harmful to agriculture such as phytopathogenic nematodes and fungi. Because most of these organisms are controlled or erradicated with toxic chemical compounds, less toxic alternative have been sought to replace them, included natural extracts, such as those of Piper. In this sense, the present work evaluated the
\end{abstract}


namaticidal and fungicidal effect of the hydroalcoholic extracts (ETOH: $\mathrm{H}_{2} \mathrm{O}$ 7: 3) of Piper lapathifolium and P. melastomoides. The nematicidal effect was determined in Panagrellus ridivivus in a 24-hour trial and the Mean Lethal Concentration $\left(\mathrm{LC}_{50}\right)$ was estimated. The fungicidal effect was evaluated in Fusarium oxysporum and $F$. solani, by the plate dilution test. The Average Effective Concentration $\left(\mathrm{EC}_{50}\right)$ was shown by the Probit model. The results showed that P. melastomoides presented a nematicidal effect at levels lower than $5 \mathrm{mg} / \mathrm{ml}$ with an LC50 of $2.32 \mathrm{mg} / \mathrm{ml}$, in the case of $P$. lapathifolium it did not present activity in the nematodes, in the case of the fungicidal activity this extract $\left(\mathrm{EC}_{50}: 26.32\right.$ and $5.83 \mathrm{mg} / \mathrm{mL}$ ), whereas $P$. melastomoides exhibited lower activity. It is concluded that $P$. melastomoides has a nematicidal effect and $P$. lapathifolium fungicide.

Keywords: Fusarium, Panagrellus redivivus, Biocidal.

\section{INTRODUCCIÓN}

El género Piper comprende cerca de 1500 especies distribuidas a lo largo del mundo en zonas tropicales, siendo más diversas en el continente americano, especialmente en bosques húmedos premontanos y tierras bajas. De los 1500 taxa descritos cerca 136 están reportadas para México y 86 para Veracruz (Sosa y Gómez-Pompa, 1994; Villaseñor, 2016). Debido a su elevada diversidad, por lo cual representa un gran potencial biológico en la búsqueda de compuestos con bioactividad. En este sentido la biotecnología, ha encontrado en las especies del género Piper un recurso natural de gran importancia, puesto que en éste. Se han reportado que tienen una amplia variedad de efectos biocidas en el género Piper, entre los que destacan la actividad bactericida, insecticida, antiprotozoarios, fungicida, antihelmíntica y nematicida (Scott et al, 2008; Carmona et al., 2016). Atribuibles a sus más de 667 fitocompuestos descritos (Parmar et al., 1997; Lee et al., 2001). Recordando que los nematodos son un problema fitosanitario para las plantaciones interés comercial, principalmente los géneros Melodoigyne y Gobodera (Johnson y Olsen, 2010); mientras que los hongos fitopatógenos son responsables de enfermedades como el marchitamiento del banano y de algunas solanáceas de importancia económica, dada su importancia se ha buscado como erradicarlos o contenerlos con métodos ecológicamente aceptables como, lo son el uso de extractos del género Piper.

\section{MATERIALES Y MÉTODOS}

\section{Colecta e identificación de especímenes}

Piper melastomoides y P. lapathifolium fueron colectados en la Reserva Ecológica la Martinica en el municipio de Banderilla, Veracruz, en enero del 2018, estos fueron identificados por comparación en los herbarios XAL y con ayuda de la base de datos del MEXU.

\section{Preparación de extractos}

$1.5 \mathrm{~kg}$ de hojas de cada especie fueron secadas a $60 \pm 5{ }^{\circ} \mathrm{C}$, posteriormente fueron molidas hasta obtener un polvo fino (Soberon et al., 2006). Los extractos fueron obtenidos mediante maceración al $10 \%$ (p:v) en Etanol $70 \%$ y concentrados a presión reducida (Kusuma et al., 2017; Moreno et al., 2000).

\section{Ensayo en Panagreullus redivisus}

La cepa de $P$. redivivus utilizada en esta investigación se cultivó en medio avena:agua (1:1). Se utilizaron nematodos J2 para los ensayos. Los gusanos se extrajeron del medio con buffer M9 (3g KH $2 \mathrm{PO}_{4}, 6$ g Na $2 \mathrm{HPO}_{4}, 5 \mathrm{~g}$ 
$\mathrm{NaCl}, 0.25 \mathrm{~g} \mathrm{MgSO}_{4} 7 \mathrm{H}_{2} \mathrm{O}$ en un litro de agua) (Brenner, 1974; Pica, 2008; Agyare et al., 2014). Los nematodos obtenidos del medio de cultivo se traspasaron a un tubo de plástico para $2 \mathrm{ml}$ y se centrifugó a $8000 \mathrm{rpm}$ por 10 minutos a temperatura ambiente, el sobrenadante se descartó y se realizaron dos lavados más con buffer M9.

El ensayo se realizó en microplacas de 96 pozos, en cada pozo se depositaban 10 organismos, para cada prueba se manejaron cinco concentraciones de los extractos resuspendidos en buffer M9, más un control sin el extracto, el diseño fue completamente al azar. El tiempo de exposición fue de 24 horas a $22 \pm$ $2^{\circ}$ C. Posteriormente se contabilizó el número de organismos muertos, todo esto se determinó mediante inspección visual con ayuda de un microscopio estereoscópico (Katiki et al., 2011; Roh y Choi, 2007; Agyare et al., 2014).

\section{Cultivo y ensayo en Fusarium spp}

Se utilizaron las cepas de Fusarium oxysporum y $F$. solani, las cuales fueron cultivas en Agar Papa Dextrosa (PDA Sigma Aldrich) a $27{ }^{\circ} \mathrm{C}$ por 7 días, después de este tiempo se mantenían en crecimiento constante y en recultivo. El ensayo se realizó mediante el método de vaciado en placa, este consistió en colocar el medio de cultivo con diferentes concentraciones de los extractos crudos, depositando en el centro de la placa de petri un disco de $5 \mathrm{~mm}$ de las cepas de Fusarium, las cajas fueron incubadas $27{ }^{\circ} \mathrm{C}$ por 7 días, posterior a esto se midió el crecimiento micelial, el diseño del experimento fue completamente al azar (Neela et al., 2014).

\section{Caracterización fitoquímica preliminar}

La identificación de los metabolitos secundarios presentes en las dos especies se realizó mediante pruebas fitoquímicas preliminares, para alcaloides (Mayer, Draguendorff y Wagner), flavonoides (Shinnoda, cloruro férrico y ácido sulfúrico), cumarinas (fluorescencia), saponinas (prueba de espuma y si esta permanecía más de 20 minutos se realizaba la prueba de Lieberman) y triterpenos y/o esteroles (Prueba de Lieberman-Bouchard), todo esto se basó en una escala cualitativa (Domínguez, 1979, Carmona-Hernández et al., 2014).

\section{Análisis estadístico}

Se determinaron los porcentajes de mortalidad para el caso de los nematodos y porcentaje de inhibición para los hongos (Finey, 1987). Para los nematodos se calculó la Concentración Letal Media $\left(\mathrm{CL}_{50}\right)$ y en los hongos la Concentración Efectiva Media ( $\left.\mathrm{CE}_{50}\right)$ se estimó mediante el modelo de regresión lineal Probit, (Finney, 1987; Jensen et al., 2006), utilizando el programa estadístico BioStat V5. Para comprobar si existía diferencias entre las concentraciones se realizó un ANOVA y una prueba posterior de Tukey, los datos no normales se analizaron una prueba ANOVA no paramétrica de Kruskal Wallis y el test posterior de Dunncan.

\section{RESULTADOS Y DISCUSIÓN}

Se determinó la actividad nematicida en uno de los dos extractos trabajados, siendo el extracto de Piper melastomoides con mayor actividad estimándose una CL50 de $2.323 \mathrm{mg} / \mathrm{mL}$ (Tabla 1) y fue el extracto más activo.Por el contrario, no se encontró efecto sobre los nematodos del extracto de $P$. lapathifolium, cuya $\mathrm{CL}_{50}$ en 3 $\mathrm{g} / \mathrm{mL}$, esto debido a que el modelo no se puede ajustar a la baja o nula mortalidad que éste presentó, la cual no difiere significativamente del control (Figura 1 a y b). Esto refuerza los ya 
reportados para algunas especies del género que han mostrado tener efecto biocida como el caso de $P$. nigrum sobre Melodoigyne incognita; $P$. chava sobre C. elegans (Wiratno et al., 2009; Atjanasuppat et al., 2009). Al igual que el extracto etanólico $P$. aduncum sobre Haemonchus contortus con una CL50 de 2.5 $\mathrm{mg} / \mathrm{mL}$. El extracto de $P$. melastomoides presentó actividad a bajas concentraciones e inclusive menores a las reportadas para algunos plaguicidas sintéticos (clorpirifos CL50 19.4 $\mathrm{mg} / \mathrm{mL}$, carbosulfan CL50 $25.3 \mathrm{mg} / \mathrm{mL}$ y deltametrina $\mathrm{CL}_{50}>40 \mathrm{mg} / \mathrm{mL}$ ) (Wiratno et al., 2009).

Tabla 1. Concentraciones letales medias y concentraciones efectivas medias de los extractos de Piper.

\begin{tabular}{llll}
\hline \multicolumn{1}{c}{ Modelo } & \multicolumn{1}{c}{ Extracto } & \multicolumn{1}{c}{ CL50 $_{\mathbf{5}} / \mathbf{C E}_{\mathbf{5 0}}$} & P \\
\cline { 2 - 4 } Panagrellus redivivus & P. melastomoides & $2.32 \mathrm{mg} / \mathrm{mL}$ & 0.016 \\
& P. lapathifolium & $3.25 \mathrm{mg} / \mathrm{mL}$ & 0.5777 \\
\hline \multirow{2}{*}{ Fusarium oxysporum } & P. lapathifolium & $26.32 \mathrm{mg} / \mathrm{mL}$ & 0.5270 \\
& P. melastomoides & $80.28 \mathrm{mg} / \mathrm{mL}$ & 0.0488 \\
Fusarium solani & P. lapathifolium & $5.83 \mathrm{mg} / \mathrm{mL}$ & 0.0001 \\
& P. melastomoides & $54.66 \mathrm{mg} / \mathrm{mL}$ & 0.0001
\end{tabular}

En el caso de la actividad fungicida se determinó que el extracto de Piper lapathifolium fue el que presentó mayor actividad sobre Fusarium solani, con una $\mathrm{CE}_{50}$ de $5.28 \mathrm{mg} / \mathrm{mL}$; con el $95 \%$ de inhibición del crecimiento (Tabla 1 y Figura 1. c, d, e, f). Para el resto de los extractos el efecto fue reducido con menos del $50 \%$ de inhibición, siendo los extractos de $P$. melastomoides los menos efectivos. Cabe mencionar que la cepa de $F$. oxysporum resultó ser menos susceptible que $F$. solani, e inclusive se ha reportado que $F$. oxysporum muestra resistencia a plaguicidas sintéticos como al carbendazim, dithane M-45 y metil tiofanato con crecimientos entre 20.5, 22.3 y $14.3 \mathrm{~mm}$ respectivamente (Gnanaskaran et al., 2015). A pesar de que los resultados no fueron los esperados para $F$. oxysporum, caso contrario a la efectividad sobre $F$. solani, además de que esto abre la posibilidad de buscar otros extractos del género Piper, con actividad fungicida puesto que se ha reportado que algunas especies como $P$. betle, tiene efectos sobre $F$. oxysporum var. vanillae, y en el caso de $P$. hispidum, $P$. eriopodom, $P$. bremedayeri, $P$. bogontense, $P$, marginatum y $P$. divacatum han probado ser efectivas sobre $F$. oxysporum var. vanillae (Tangarife-Castaño et al., 2014; Singha et al., 2011; Ngurah et al., 2007). Por ello se deben ampliar los estudios en búsqueda de extractos que presenten mayor actividad. El análisis fitoquímico preliminar dio como resultados la presencia de alcaloides, terpenos y flavonoides, así como nula presencia de saponinas y cumarinas (Tabla 2). 
Carmona et al., 2018

Tabla 2. Análisis fitoquímico preliminar de las dos especies de Piper.

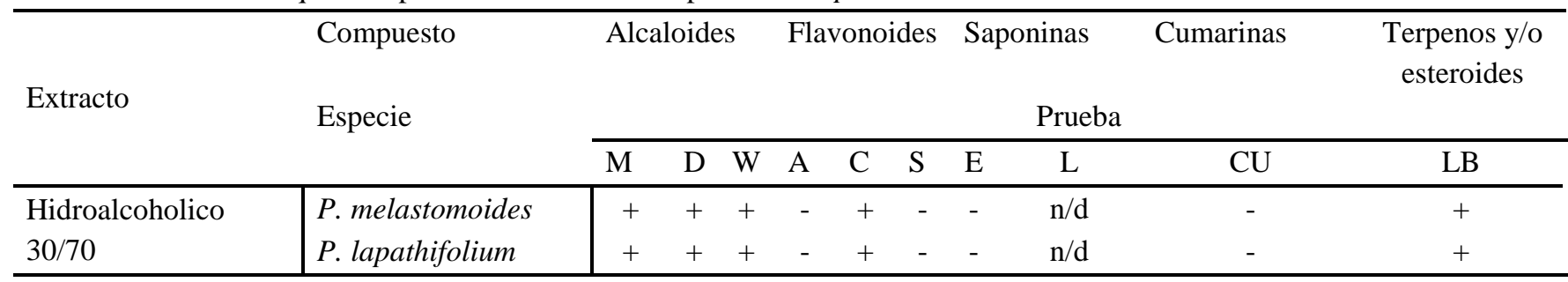

Alcaloides: $\mathrm{M}=$ Prueba de Mayer, $\mathrm{D}=$ prueba de Drangendorff y $\mathrm{W}=$ prueba de Wagner. Flavonoides: $\mathrm{A}=$ Prueba del $\mathrm{H}_{2} \mathrm{SO}_{4}, \mathrm{C}=\mathrm{FeCL}_{2}, \mathrm{~S}=$ prueba de Shinoda. Saponinas: $\mathrm{E}=$ Prueba de la espuma, $\mathrm{L}=$ Prueba de Lieberman. Cumarinas: $\mathrm{CU}=$ Prueba de Fluorescencia. Triterpenos y/o Esteroles: LB= Prueba de Lieberman-Bouchard. Intensidad: Presencia +, Ausencia $-\mathrm{y} \mathrm{n} / \mathrm{d}$ no determinada.

A

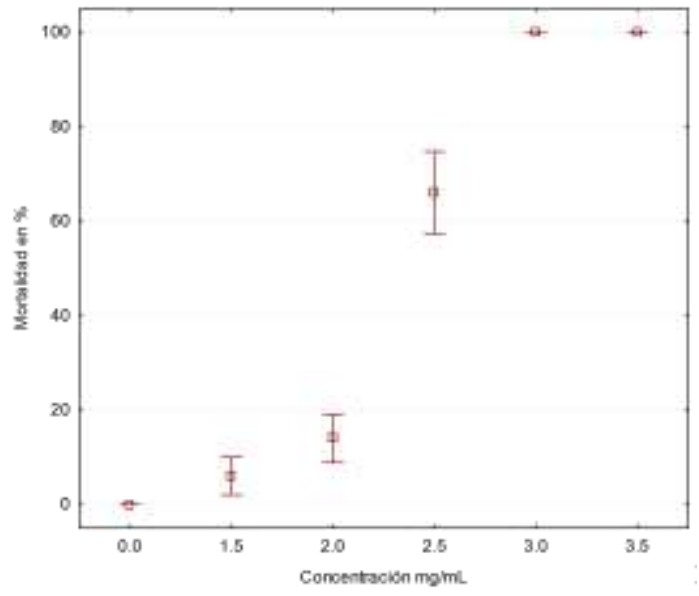

$\mathrm{C}$

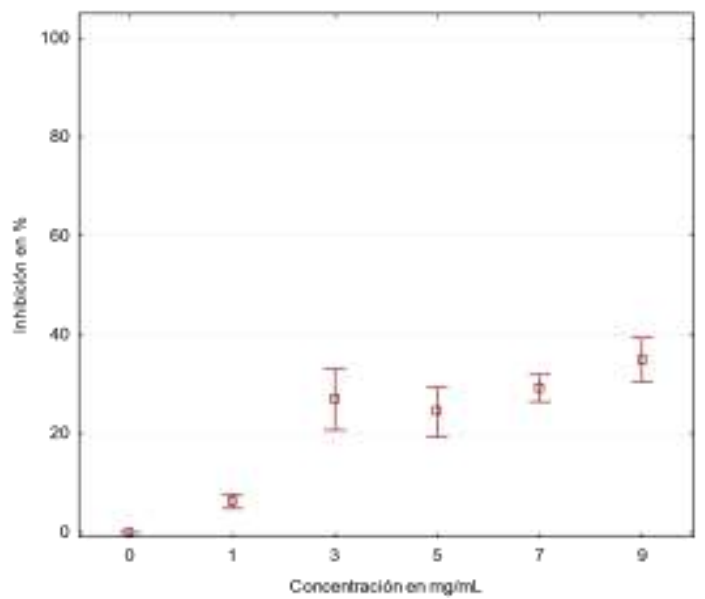

$\mathrm{E}$
B

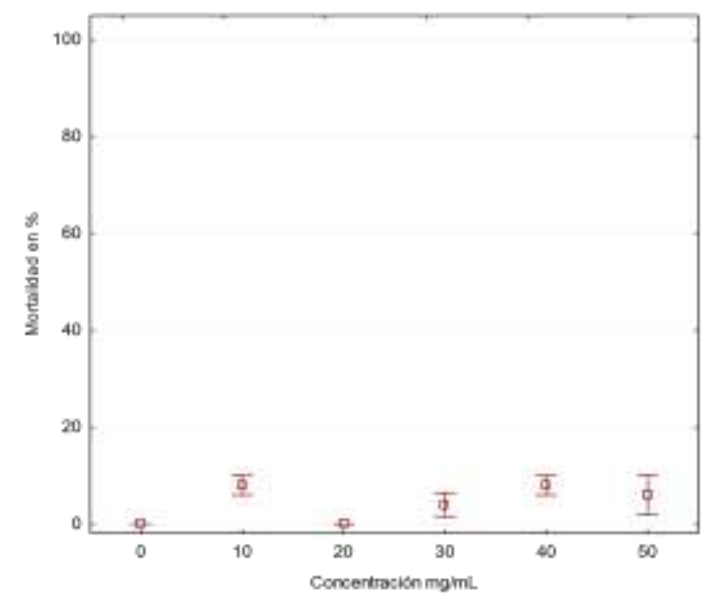

D

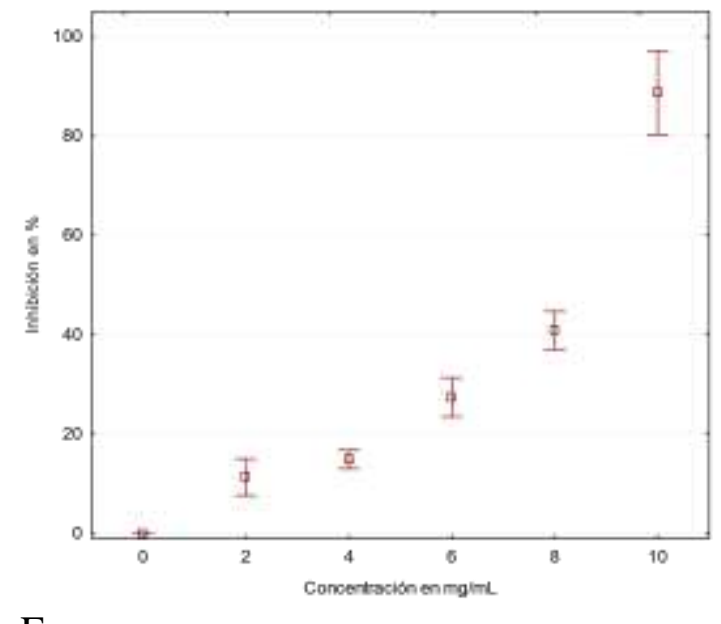



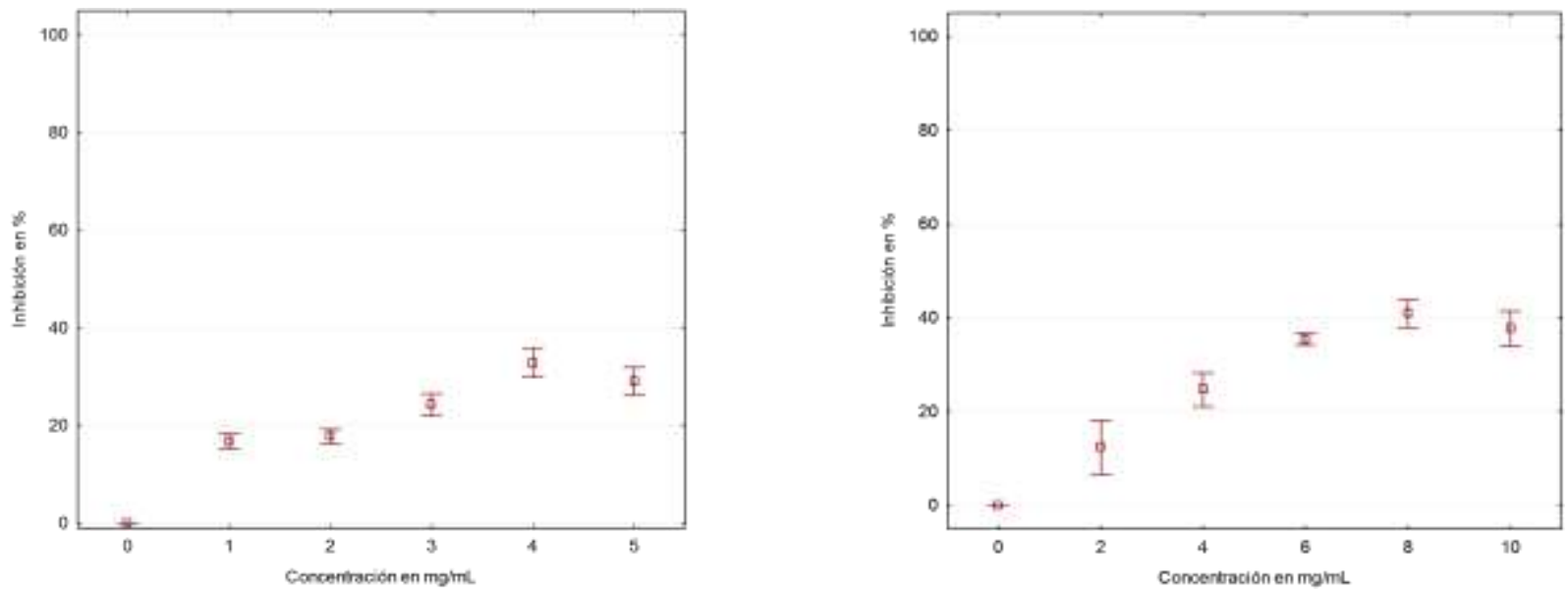

Figura 1. Porcentaje de mortalidad de los extractos de Piper lapathifolium (a) y de P. melastomoides (b) en Panagrellus redivivus, porcentaje de inhibición de P. lapathifolium en Fusarium oxysporum(c) y $F$. solani (d), (e) porcentaje inhibición de P. melastomoides en $F$. oxysporum y (f) en $F$. solani

\section{CONCLUSIONES}

El efecto nematicida solo en encontró en el extracto de Piper melastomoides, y fue nulo en P. lapathifolium. La actividad en Fusarium oxysporum fue menor al $50 \%$ tanto para $P$. lapathifolium como para $P$. melastomoides, en cambio se encontró mayor inhibición del crecimiento con el extracto de $P$. lapathifolium en F. solani.

\section{LITERATURA CITADA}

Agyare, C., Spiegler, V., Sarkodie, H., Asase, A., Liebau, E. y Hensel, A. 2014. An ethnopharmacological survey and in vitro confirmation of the ethnopharmacological use of medicinal plants as anthelmintic remedies in the Ashanti region, in the central part of Ghana. Journal of Ethnopharmacology 158 PART A: 255-263.

https://doi.org/10.1016/j.jep.2014.10.029
Atjanasuppat, K., Wongkham, W., Meepowpan, P., Kittakoop, P., Sobhon, P., Bartlett, A. y Whitfield, P. J. 2009. In vitro screening for anthelmintic and antitumour activity of ethnomedicinal plants from Thailand. Journal of Ethnopharmacology 123(3): 475-482. https://doi.org/10.1016/j.jep.2009.03.010

Brenner, S. 1974. The genetics of Caenorhabditis elegans. Genetics 77(1): 71-94.

https://doi.org/10.1093/genetics/77.1.71

Carmona-Hernández, Ó., del Socorro Fernández, M., Palmeros-Sánchez, B. y Lozada-García, J. A. 2014. Actividad insecticida de extractos etanólicos foliares de nueve piperáceas (Piper spp.) en Drosophila melanogaster. Revista Internacional de Contaminación Ambiental 30: 67-73. 
Carmona-Hernández, O., Lozada-García, J. A., Martínez-Hernández, M. J., Fernández, M. S. y Torres-Pelayo, V. R. 2016. Piper L. genus potential as natural biocide. Wulfenia Journal 23(6): 65-95.

Domínguez, A. X. 1979. Métodos de Investigación Fitoquímica. Editorial Limusa, México, D.F.

Finney, D. L. 1987. Chapter 18: Assay base don quantal responses. Statistical Method in Biological Assay 469-490.

Juárez-Becerra, G. P., Sosa-Morales, M. E. y López-Malo, A. 2010. Hongos fitoptógenos de alta importancia económica: descripción y métodos de control. Temas Selectos de Ingeniería de Alimentos 4(2): 14-23.

Jensen, H. R., Scott, I. M., Sims, S., Trudeau, V. L. y Arnason, J. T. 2006. Gene expression profiles of Drosophila melanogaster exposed to an insecticidal extract of Piper nigrum. Journal of Agricultural and Food Chemistry 54(4): 1289-1295. https://doi.org/10.1021/jf052046n

Johnson, S. B., Olsen, N., Rosen, C. y Spooner, D. M. 2010. Commercial potato production in North America. American Journal of Potato Research 87 suppl: 190.

Katiki, L. M., Ferreira, J. F. S., Zajac, A. M., Masler, C., Lindsay, D. S., Chagas, A. C. S. y Amarante, A. F. T. 2011. Caenorhabditis elegans as a model to screen plant extracts and compounds as natural anthelmintics for veterinary use. Veterinary Parasitology 182(2-4): 264268.

https://doi.org/10.1016/j.vetpar.2011.05. 020
Kusuma, S. A. F., Tjitraresmi, A. y Susanti, G. 2017. Antibacterial effect of red Piper betel leaf (Piper crocatum ruiz \& amp; pav.) ethanol extracts to Lactobacillus acidophilus and $L$. bifidus growth inhibition. Asian Journal of Pharmaceutical and Clinical Research 10(14): https://doi.org/10.22159/ajpcr.2017.v10s 2.19490

Lee, S. E., Park, B. S., Kim, M. K., Choi, W. S., Kim, H. T., Cho, K. Y., Lee. S. G. y Lee, H. S. 2001. Fungicidal activity of pipernonaline, a piperidine alkaloid derived from long pepper, Piper longum L., against phytopathogenic fungi. Crop Protection 20(6): 523-528.https://doi.org/10.1016/S0261-2 194(00)00172-1

Moreno, M. E., González, S., Acevedo, L., Morales, G., Betancur, M, López, J. J. y Peláez, C. A. 2000. Drosophila melanogaster (Diptera: Drosophilidae): modelo biológico para la estandarización de extractos naturales con actividad insecticida (El NeemAzadirachta indica- un caso particular). Revista Entomológica de Colombia 26 (1-2): 21-55.4

Neela, F. A., Sonia, I. A. y Shamsi, S. 2014. Antifungal activity of selected medicinal plant extract on Fusarium oxysporum Schlecht the causal agent of fusarium wilt disease in tomato. American Journal of Plant Sciences 05(18): 26652671.

https://doi.org/10.4236/ajps.2014.518281 
Ngurah, D. y Oshawa, K. 2007. Fungicidal activity of Piper betle extract against Fusarium oxysporum f. sp. Vanilla. JISSAAS 13(2): 40-46.

Parmar, V. S., Jain, S. C., Bisht, K. S., Jain, R., Taneja, P., Jha, A., Om, D., Tyagi, A. K., Prasad, J., Wengel, T C., Olsen, E. y Per, M. B. Phytochemistry of genus Piper. Phytochemistry 46(4): 597-673. https://doi.org/10.1016/S0031-9422(97)0 0328-2

Pica, G. Y. 2008. Ensayo de toxicidad con el nemátodo Panagrellus redivivus. Ensayos Toxicológicos para la Evaluación de Sustancias Químicas en Agua y Suelo 139-154

Roh, J. Y. y Choi, J. 2008. Ecotoxicological evaluation of chlorpyrifos exposure on the nematode Caenorhabditis elegans. Ecotoxicology and Environmental Safety 71(2): 483-489. https://doi.org/10.1016/j.ecoenv.2007.11. 007

Scott, I. M., Jensen, H. R., Philogène, B. J. R. y Arnason, J. T. 2008. A review of Piper spp. (Piperaceae) phytochemistry, insecticidal activity and mode of action. Phytochemistry Reviews 7(1): 65-75. https://doi.org/10.1007/s11101-006-9 058-5

Singha, I. M., Kakoty, Y., Unni, B. G., Kalita, M. C., Das, J., Naglot, A., Wann S. B. y Singh, L. 2011. Control of Fusarium wilt of tomato caused by Fusarium oxysporum f. sp. lycopersici using leaf extract of Piper betle L.: A preliminary study. World Journal of Microbiology and Biotechnology 27(11): 2583-2589. https://doi.org/10.1007/s1 1274-011-0730 $-6$
Soberón, G. V., Rojas, C., Saavedra, J., Kato, M. J. y Delgado, G. E. 2006. Acción biocida de plantas de Piper tuberculatum Jacq. sobre Diatrea saccharalis (Lepidóptera, Pyralidae). Revista Peruana de Biología 13(1): 107112.

https://doi.org/10.15381/rpb.v13i1.1770

Sosa, V. y Gómez-Pompa, A. 1994. Lista florística del estado de Veracruz. Flora de Veracruz 82: 1-245.

Tangarife-Castaño, V., Correa-Royero, J. B., Roa-Linares, V. C., Pino-Benitez, N., Betancur-Galvis, L. A., Durán, D. C., Staschenko, E. E. y Mesa-Arango, A. C. 2014. Anti-dermatophyte, anti-Fusarium and cytotoxic activity of essential oils and plant extracts of Piper genus. Journal of Essential Oil Research 26(3): 221-227.

https://doi.org/10.1080/10412905.2014.8 82279

Varsha, H. y Sonali, S. 2014. Studies on qualitative phytochemical analysis of selected species of Piper. International Journal of Life Sciences Special Issue A2: $156-158$

Villaseñor, J. L. 2016. Checklist of the native vascular plants of Mexico. Revista Mexicana de Biodiversidad 87(3): 559902.

https://doi.org/10.1016/j.rmb.2016.06.01 7

Wiratno, T. D., Van den Berg, H., Riksen, J. A. G., Rietjens, I. M. C. M., Djiwanti, S. R. y Murk, A. J. 2009. Nematicidal activity of plant extracts against the root-knot nematode, Meloidogyne incognita. The Open Natural Products Journal 2:77-85. https://doi.org/10.2174/1874848100902 010077 
Copyright (c) 2018 Oscar Carm ona Hernández, José Armando Lozada G arcia, Maria del Socorro Fernández, Mauricio Luna Rodriguez, Maria de Jesús Martinez Hernández y José Antonio Guerrero Analco

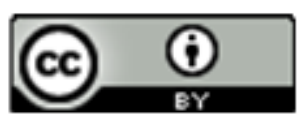

Este tex to está protegido por una licencia CreativeCommons 4.0 .

Usted es libre para Compartir —copiar y redistribuir el $\mathrm{m}$ aterial en cualquier medio o formato- $\mathrm{y}$ Adaptar el documento - remezclar, transformar y crear a partir del material- para cualquier propósito, incluso para fines comerciales, siempre que cumpla la condición de:

Atribución: Usted debe dar crédito a la obra original de manera adecuada, proporcionar un enlace a la licencia, e indicar si se han realizado cambios. Puede hacerlo en cualquier forma razonable, pero no de forma tal que sugiera que tiene el apoyo del licenciante o lo recibe por el uso que hace de la obra.

Resumenclelicencia - Textocompletodelalicencia 